\title{
Braid Matrices and Structure Constants for Minimal Conformal Models
}

\author{
G. Felder ${ }^{1 \star \star \star \star}$ J. Fröhlich ${ }^{2}$ and G. Keller ${ }^{2}$ \\ ${ }^{1}$ School of Mathematics, The Institute for Advanced Study, Princeton, NJ 08540, USA \\ 2 Theoretische Physik, ETH-Hönggerberg, CH-8093 Zürich, Switzerland
}

\begin{abstract}
Using the Feigin-Fuchs representation of minimal conformal models in a form introduced recently by one of us, the braid group representation matrices, describing the analytic continuation properties of conformal blocks, are computed. In a suitable normalization, their matrix elements are shown to essentially factorize into pairs of Boltzmann weights of critical RSOS models in a certain limit of the spectral parameter. These Boltzmann weights are related to quantum group $R$-matrices by the vertex-SOS transformation. We show that the crossing symmetry of the four-point function in left-right symmetric models follows from a quantum group relation, also called crossing symmetry. This observation gives a simple way to evaluate the structure constants.
\end{abstract}

\section{Introduction}

In recent times, much study was devoted to the connection between conformal field theory and representations of the braid group [1-11]. The reasons for this interest are two-fold: First, understanding the braid group representation carried by the conformal blocks is necessary to complete the conformal bootstrap program [12] in the general case. Second, this method, possibly in connection with modular invariance, might ultimately lead to a classification of two-dimensional conformal theories, at least of rational theories.

Some time ago, Tsuchiya and Kanie [1] found a connection between the braid matrices describing the monodromy of conformal blocks of the fundamental field in the $S U(2)$ WZW model and the Temperley-Lieb-Jones algebra. The same structure was seen to arise [4] for the braid matrices of the $\phi_{(12)}$ field in minimal models [12]. These matrices are connected by the vertex-SOS transformation to the spin $\frac{1}{2} R$-matrices of exactly solvable vertex models in a certain limit of the parameters. By the fusion procedure, $R$-matrices corresponding to higher spin

\footnotetext{
* Supported by NSF grant DMS 8610730

$\star \star$ Address after March 1989: Theoretische Physik, ETH-Hönggerberg, 8093 Zürich, Switzerland
} 
representations were constructed ([13] and references therein). In the quantum group [14-17] language, these $R$-matrices appear as generators of the commutant in a tensor product representation of the quantum group $U_{q}(S U(2))$.

Since higher primary fields are constructed out of fundamental fields by a fusion procedure, it has been conjectured $[1,4,18]$ that braid matrices of the higher spin fields in the $S U(2) \mathrm{WZW}$ model are given by $R$-matrices corresponding to higher spin, and that braid matrices of primary fields $\phi_{\left(n^{\prime}, n\right)}$ of minimal model factorize into pairs of $S U(2) \mathrm{WZW}$ braid matrices.

In the first part of this paper, we compute the braid matrices explicitly for what we call Dotsenko-Fateev models [19-21]. These models have a Feigin-Fuchs representation and, at rational values of a complex parameter $\alpha_{-}^{2}$, they reduce to minimal models [12]. We show that the braid matrix elements essentially factorize into two factors corresponding to quantum group representations with quantum group parameters $q=\exp 2 \pi i \alpha_{-}^{2}$ and $q=\exp 2 \pi i \alpha_{-}^{-2}$.

A peculiarity of braid group representations arising from quantum group representations is that they produce knot invariants [21]. This has recently led to the proposal [22] that rational conformal theories should be understood in terms of three-dimensional generally covariant theories.

In the second part of this paper, we show, in the example of minimal and Dotsenko-Fateev models, that an essential property of conformal field theory, namely crossing symmetry, follows from a quantum group relation also called crossing symmetry. The latter property is essential for the application to knot theory. It would be interesting to understand it from a three-dimensional point of view. We also show how this relation can be used to compute structure constants and prove the consistency of the solution.

The last part of this paper is devoted to the minimal model limit $\alpha_{-}^{2} \rightarrow p / p^{\prime}$, where some care has to be taken to avoid singularities of braid matrix elements.

The class of models we consider in this paper consists of the Dotsenko-Fateev (DF) models [19] parametrized by a complex number $\alpha_{-}^{2}$, related to the central charge of the Virasoro algebra by $c=13-6 \alpha_{-}^{2}-6 \alpha_{-}^{-2}$. For generic $\alpha_{-}^{2}$, these models are neither rational nor unitary and the interesting limit is when $\alpha_{-}^{2}$ tends to a positive rational number $p / p^{\prime}$. In this limit one recovers the minimal models of [12]. If $\left|p-p^{\prime}\right|=1$ and $\min \left(p, p^{\prime}\right) \geqq 3$ [23], minimal models are unitary.

For generic $\alpha_{-}^{2}$, the space of states of a DF model is a direct sum of irreducible representation spaces for a pair of Virasoro algebras

$$
\mathscr{H}=\bigoplus_{I} \mathscr{H}_{I} \otimes \mathscr{H}_{I} \text {. }
$$

The sum runs over all degenerate highest weight representations with given central charge. The label $I$ stands for a pair $\left(i^{\prime}, i\right)$ of positive integers and the corresponding highest weight is

$$
h_{I}=h_{\left(i^{\prime} i\right)}=\frac{1}{4}\left(i^{\prime 2}-1\right) \alpha_{-}^{2}-\frac{1}{2}\left(i^{\prime} i-1\right)+\frac{1}{4}\left(i^{2}-1\right) \alpha_{-}^{-2} .
$$

Primary field operators of weight $h_{J}$ split into holomorphic and antiholomophic components

$$
\Phi_{J}(z, \bar{z})=\sum_{I K} D_{J K}^{I} V_{J K}^{I}(z) \otimes V_{J K}^{I}(\bar{z})
$$


The component $V_{J K}^{I}(z)$ maps $\mathscr{H}_{K}$ to $\mathscr{H}_{I}$ and (if non-zero) is determined, up to normalization, by its commutation relations with Virasoro generators. In the case of minimal models the sum in (1.1) becomes a finite one: $\left(i^{\prime} i\right)$ run over $1 \leqq i^{\prime} \leqq p^{\prime}-1$, $1 \leqq i \leqq p-1$, and there are finitely many primary fields.

In [24] a new version of the Feigin-Fuchs representation was given: the spaces $\mathscr{H}_{I}$ were identified with cohomology groups of a certain BRST operator on Fock spaces and chiral primary fields $V_{J K}^{I}(z)$ act as BRST invariant "screened" free field vertex operators. We compute the braid matrices in this formalism. We first show how to construct general screened vertex operators by fusion of "fundamental" fields. This gives an expression for braid matrices for general fields in terms of fundamental braid matrices. The latter are then computed by contour deformation. We note here that some of the braid matrices were computed in [25] in a different setting, to compute all structure constants.

A similar analysis could be done for more general models such as the Fateev-Lykyanov models [26]. What is missing in that case is the theory of the structure of Fock spaces as modules over extended algebras.

Finally, we note that DF models have also been studied in the context of the quantum Liouville theory [27].

\section{Fusion and Braid Matrices of Screened Vertex Operators}

In [24] chiral primary fields of DF models were expressed in terms of screened vertex operators

$$
V_{n^{\prime} n}^{r^{\prime}}(z)=\int V_{\alpha_{n^{\prime} n}}(z) V_{\alpha_{-}}\left(u_{1}^{\prime}\right) \cdots V_{\alpha_{-}}\left(u_{r^{\prime}}^{\prime}\right) V_{\alpha_{+}}\left(u_{1}\right) \cdots V_{\alpha_{+}}\left(u_{r}\right) \prod_{i^{\prime}, i} d u_{i^{\prime}}^{\prime} d u_{i}
$$

with charges $\alpha_{n^{\prime} n}=\frac{1}{2}\left(1-n^{\prime}\right) \alpha_{-}+\frac{1}{2}(1-n) \alpha_{+}, \alpha_{+} \alpha_{-}=-1$. The operator $V_{\alpha}(z)$ is the familiar Wick-ordered exponential of the free field

$$
T_{\alpha} z^{\alpha a_{0}} \exp \left(\alpha \sum_{1}^{\infty} a_{-n} z^{n} / n\right) \exp \left(-\alpha \sum_{1}^{\infty} a_{n} z^{-n} / n\right),
$$

$\left[a_{n}, a_{m}\right]=2 n \delta_{n,-m},\left[a_{n}, T_{\alpha}\right]=2 \delta_{n, 0} \alpha T_{\alpha}$. The contours of integration, $C_{i}^{\prime}$ of $u_{i}^{\prime}$ and $C_{i}$ of $u_{i}$, are depicted in Fig. 1. Since the integrand is many-valued, we must specify a convention to make it uniquely defined. We require that the expectation value of the integrand between highest weight states be real when the variables are ordered on the positive real axis $\left(0<u_{r}<\cdots<u_{1}<u_{r^{\prime}}^{\prime}<\cdots<u_{1}^{\prime}<z\right)$ and the charges are real. The value of $(2.1)$ is then unambiguously defined if we give a path of analytic continuation in $\mathbb{C}^{r+r^{\prime}+1}-\bigcup_{\alpha<\beta}\left\{z_{\alpha}=z_{\beta}\right\}$, going from a point where the variables are ordered on $\mathbb{R}^{+}$to a point on the integration domain. This path is represented by a dashed line in Fig. 1.

The precise definition of screened vertex operators involves an analytic continuation from a region of $\alpha_{-}^{2}$-plane where the integrals in (2.1) converge. This region is given by [20]

$$
-\frac{1}{2}<\operatorname{Re} \alpha_{ \pm}^{2}<0 .
$$

Since $\alpha_{+} \alpha_{-}=-1$, we see that this region does not contain any real points. We 


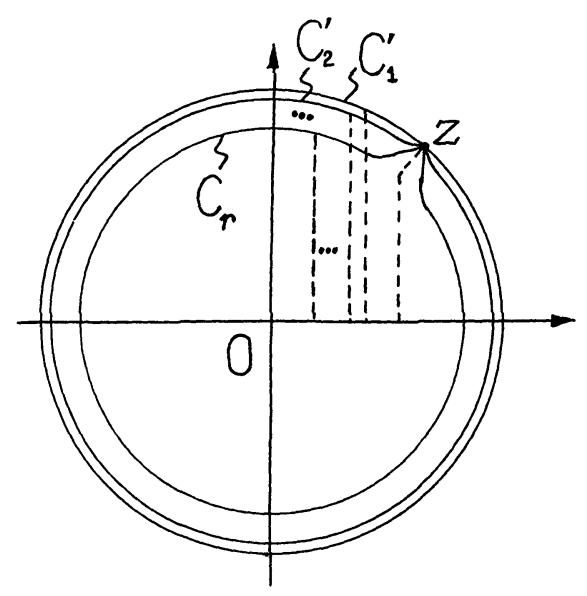

Fig. 1. The contours of integration $C_{i}^{\prime}$ of $u_{i}^{\prime}$ and $C_{i}$ of $u_{i}$ in (2.1). The dashed lines represent the analytic continuation paths

compute the braid matrices in this region and then extend the results by analytic continuation in $\alpha^{2}$.

The braid matrices can be computed recursively in terms of "elementary" braid matrices by using "fusion":

$$
\begin{aligned}
& V_{n^{\prime}+1, n}^{r^{\prime}, r}(z)=\lim _{\zeta \rightarrow z}(\zeta-z)^{-2 \alpha_{21} \alpha_{n^{\prime} n}} V_{21}^{00}(\zeta) V_{n^{\prime} n}^{r^{\prime} r}(z), \\
& V_{n^{\prime}+1, n}^{r^{\prime}+1, r}(z)=e^{-\pi i 2 \alpha_{-} \alpha_{n^{\prime} n}} \lim _{\zeta \rightarrow z}(\zeta-z)^{-2 \alpha_{21} \alpha_{n^{\prime} n}} V_{21}^{10}(\zeta) V_{n^{\prime} n}^{r^{\prime} r}(z), \\
& V_{n^{\prime}, n+1}^{r^{\prime} r}(z)=\lim _{\zeta \rightarrow z}(\zeta-z)^{-2 \alpha_{12} \alpha_{n^{\prime} n}} V_{12}^{00}(\zeta) V_{n^{\prime} n}^{r^{\prime} r}(z), \\
& V_{n^{\prime}, n+1}^{r^{\prime}, r+1}(z)=e^{-\pi i 2 \alpha_{+} \alpha_{n^{\prime} n}} \lim _{\zeta \rightarrow z}(\zeta-z)^{-2 \alpha_{12} \alpha_{n^{\prime} n}} V_{12}^{01}(\zeta) V_{n^{\prime} n}^{r^{\prime} r}(z) .
\end{aligned}
$$

The phase in (2.3b) comes from changing the reference point for the analytic continuation from $0<u_{r}<\cdots<u_{1}^{\prime}<z<u^{\prime}<\zeta$ to $0<u_{r}<\cdots<u_{1}^{\prime}<u^{\prime}<z<\zeta, u^{\prime}$ being the integration variable in $V_{21}^{10}$. The same applies to the phase in $(2.3 \mathrm{~d})$. Equations (2.3) are valid in the region of complex $\alpha_{-}^{2}$-plane described above. They follow from the standard operator product expansion of vertex operators. The crucial point is that the contours over which vertex operators with charge $\alpha_{+}$and $\alpha_{\text {- }}$ are integrated can be interchanged without changing the value of the integral (2.1), as noted in [20] in a slightly different language. The reason for this is that the difference between (2.1) and the integral with, say, the $u_{1}$-contour and $u_{r^{\prime}}^{\prime}$-contour interchanged contains a term ${ }^{1}$

$$
\oint_{u_{1}} V_{\alpha_{-}}\left(u_{r^{\prime}}^{\prime}\right) V_{\alpha_{+}}\left(u_{1}\right) d u_{r^{\prime}}^{\prime}=2 \pi i \frac{\alpha_{-}}{\alpha_{+}+\alpha_{-}} \frac{\partial}{\partial u_{1}} V_{\alpha_{-}+\alpha_{+}}\left(u_{1}\right)
$$

which, being integrated over $u_{1}$, gives a vanishing contribution.

$\oint_{u_{1}}$ is the integration over a small circle surrounding $u_{1}$ 
It is convenient to introduce the notation

$$
V_{\left(n^{\prime} n\right)\left(m^{\prime} m\right)}^{\left(l^{\prime} l\right)}(z)=e^{i \theta\left(n^{\prime} n\right)\left(m^{\prime} m\right)} V_{n^{\prime} n}^{r^{\prime} r}(z)
$$

to indicate vertex operators mapping BRST states in the Fock space $F_{m^{\prime} m}$ to BRST states in $F_{l^{\prime} l}$. The relation between the number of screening operators $r^{\prime}, r$ and the representation labels is given by charge conservation: $l^{\left({ }^{\prime}\right)}=n^{\left({ }^{\prime}\right)}+m^{\left({ }^{\prime}\right)}-2 r^{\left({ }^{\prime}\right)}-1$. In Appendix A we review and extend the results of [24] on the BRST cohomology of Fock spaces. The space of BRST states in the Fock space $F_{m^{\prime} m}$ is called $\mathscr{H}_{m^{\prime} m}$. It is irreducible under the Virasoro algebra. By construction, see (2.3), we always have $0 \leqq r^{\left({ }^{\prime}\right)} \leqq n^{\left({ }^{\prime}\right)}-1$ in (2.5). Moreover, since $V_{(12)\left(l^{\prime} 1\right)}^{\left(l^{\prime}\right)}$ and $V_{(21)(1 l)}^{(0 l)}$ vanish identically on BRST states for all $l^{\prime}, l \geqq 1$ (See Appendix A), we conclude that all non-vanishing operators (2.5) constructed by fusion (2.3) map $\mathscr{H}_{m^{\prime} m}$ with $m^{\prime}, m \geqq 1$ to $\mathscr{H}_{l^{\prime} l}$ with $l^{\prime}, l \geqq 1$. A more detailed analysis [24] shows that $V_{\left(n^{\prime} n\right)\left(m^{\prime} m\right)}^{\left(l^{\prime} l\right)}$ vanishes on BRST states for generic $\alpha_{-}^{2}$ unless

$$
\begin{aligned}
& \left|n^{\left({ }^{\prime}\right)}-m^{\left({ }^{\prime}\right)}\right|+1 \leqq l^{(\prime)} \leqq n^{\left({ }^{\prime}\right)}+m^{\left({ }^{\prime}\right)}-1, \\
& n^{\left({ }^{\prime}\right)}+m^{(\prime)}+l^{\left({ }^{\prime}\right)}+1 \leqq 0 \bmod 2 .
\end{aligned}
$$

These fusion rules are more severe if $\alpha_{-}^{2}$ is rational as will be discussed in Sect. 4 . The phase

$$
\begin{aligned}
\theta_{\left(n^{\prime} n\right)\left(m^{\prime} m\right)}^{\left(l^{\prime} l\right)}= & \frac{\pi}{2}\left\{\left(2 n^{\prime}+1\right) r+(2 n+1) r^{\prime}\right\} \\
& -\pi \alpha_{-}^{2} r^{\prime}\left(r^{\prime}-m^{\prime}\right)-\pi \alpha_{+}^{2} r(r-m)
\end{aligned}
$$

was introduced to simplify further calculations. This phase makes the operator on the left-hand side of (2.4) a real operator for $z, \alpha_{-}^{2}$ real, in the sense that all its matrix elements are real in a basis given by monomials of Virasoro generators acting on the highest weight state. Relations (2.3) become

$$
\begin{aligned}
& V_{\left(n^{\prime}+1, n\right)\left(m^{\prime} m\right)}^{\left(l^{\prime} l\right)}(z)=(-1)^{1 / 2(n+m-l-1)} \lim _{\zeta \rightarrow z}(\zeta-z)^{-2 \alpha_{21} \alpha_{n^{\prime} n}} V_{(21)\left(l^{\prime} \pm 1, l\right)}^{\left(l^{\prime} l\right)}(\zeta) V_{\left(n^{\prime} n\right)\left(m^{\prime} m\right)}^{\left(l^{\prime} \pm 1, l\right)}(z), \\
& V_{\left(n^{\prime} n+1\right)\left(m^{\prime} m\right)}^{\left(l^{\prime} l\right)}(z)=(-1)^{1 / 2\left(n^{\prime}+m^{\prime}-l^{\prime}-1\right)} \lim _{\zeta \rightarrow z}(\zeta-z)^{-2 \alpha_{12} \alpha_{n^{\prime} n} n} V_{\left(1^{\prime}\right)\left(l^{\prime} l \pm 1\right)}^{\left(l^{\prime} l\right)}(\zeta) V_{\left(n^{\prime} n\right)\left(m^{\prime} m\right)}^{\left(l^{\prime}, l \pm 1\right)}(z) .
\end{aligned}
$$

We often abbreviated $L=\left(l^{\prime}, l\right), N=\left(n^{\prime}, n\right)$, etc. in the following. The braid matrices are defined by the equation

$$
V_{M B}^{A}(z) V_{N C}^{B}(w)=\sum_{D} R(A, M, N, C)_{B D} V_{N D}^{A}(w) V_{M C}^{D}(z) .
$$

The left-hand side of this equation, valid for $|w|>|z|$, is understood as analytic continuation from the region $|w|<|z|, 0 \leqq \arg z$, $\arg w<2 \pi$ along a path such that $z$ circumvents $w$ counterclockwise. The braid matrices can be computed using the following basic identity for vertex operators:

$$
V_{\alpha}(z) V_{\beta}(w)=e^{\pi i 2 \alpha \beta} V_{\beta}(w) V_{\alpha}(z),
$$

with same convention as in (2.8). 
The fusion relations (2.7) imply the following recursion relations for braid matrices (as before $A=\left(a^{\prime}, a\right)$ etc.):

$$
R(A, M+(1,0), N, C)_{B D}=(-1)^{1 / 2(a-b+c-d)} \sum_{D_{1}} R\left(A(21) N D_{1}\right)_{A_{1} D} R\left(A_{1} M N C\right)_{B D_{1}},
$$

$$
R(A, M+(0,1), N, C)_{B D}=(-1)^{1 / 2\left(a^{\prime}-b^{\prime}+c^{\prime}-d^{\prime}\right)} \sum_{D_{1}} R\left(A(12) N D_{1}\right)_{A_{1} D} R\left(A_{1} M N C\right)_{B D_{1}}
$$

$$
R(A, M, N+(1,0), C)_{B D}=(-1)^{1 / 2(a-b+c-d)} \sum_{A_{1}} R\left(A M(21) B_{1}\right)_{B A_{1}} R\left(A_{1} M N C\right)_{B_{1} D}
$$

$$
R(A, M, N+(0,1), C)_{B D}=(-1)^{1 / 2\left(a^{\prime}-b^{\prime}+c^{\prime}-d^{\prime}\right)} \sum_{A_{1}} R\left(A M(12) B_{1}\right)_{B A_{1}} R\left(A_{1} M N C\right)_{B_{1} D}
$$

These equations, represented in Fig. 2, allow us to compute braid matrices for arbitrary $M, N$ in terms of braid matrices involving the fields $(1,2)$ and $(2,1)$ only. We proceed to calculate the latter matrices. Although this computation may be done by exploiting the fact that the four-point function obeys a hypergeometric differential equation $[27,12]$, it is instructive to do it directly in this formalism.

Consider the product of two $(2,1)$ fields mapping $\mathscr{H}_{L}$ to $\mathscr{H}_{L^{\prime}}$. The possible values for $L^{\prime}$ are $L+(2,0), L, L-(2,0)$. In the first case these fields are represented by vertex operators with no screening charge, and the braid matrix is just a phase

$$
R(L+(2,0),(2,1),(2,1), L)_{L+(1,0), L+(1,0)}=e^{i \pi \alpha_{-}^{2} / 2} .
$$

Similarly,

$$
R(L+(0,2),(1,2),(1,2), L)_{L+(0,1), L+(0,1)}=e^{i \pi \alpha_{+}^{2} / 2} .
$$

In the second case there is a screening operator, that may be attached to either of the two fields. By deforming the contour of integration we can write both sides of (2.8) in terms of the integrals

$$
I_{1,2}(z, w)=\int_{\gamma_{1,2}} V_{\alpha_{21}}(w) V_{\alpha_{-}}(u) V_{\alpha_{21}}(z) d u,
$$

where $\gamma_{1,2}$ are contours drawn in Fig. 3 .
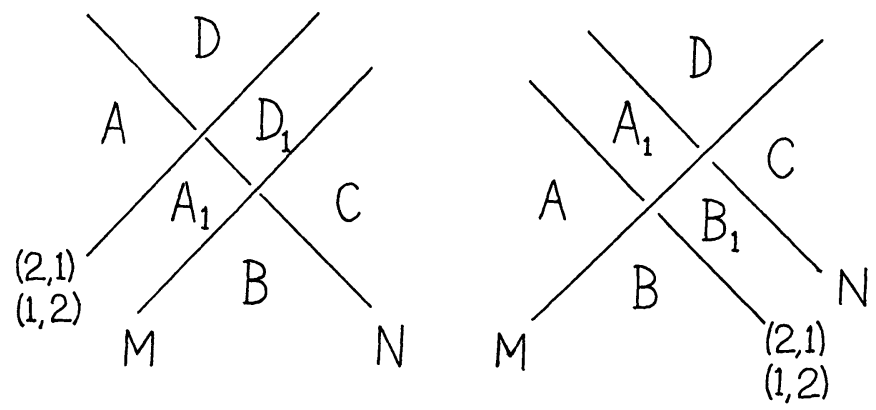

Fig. 2. Pictorial representation of (2.10) 

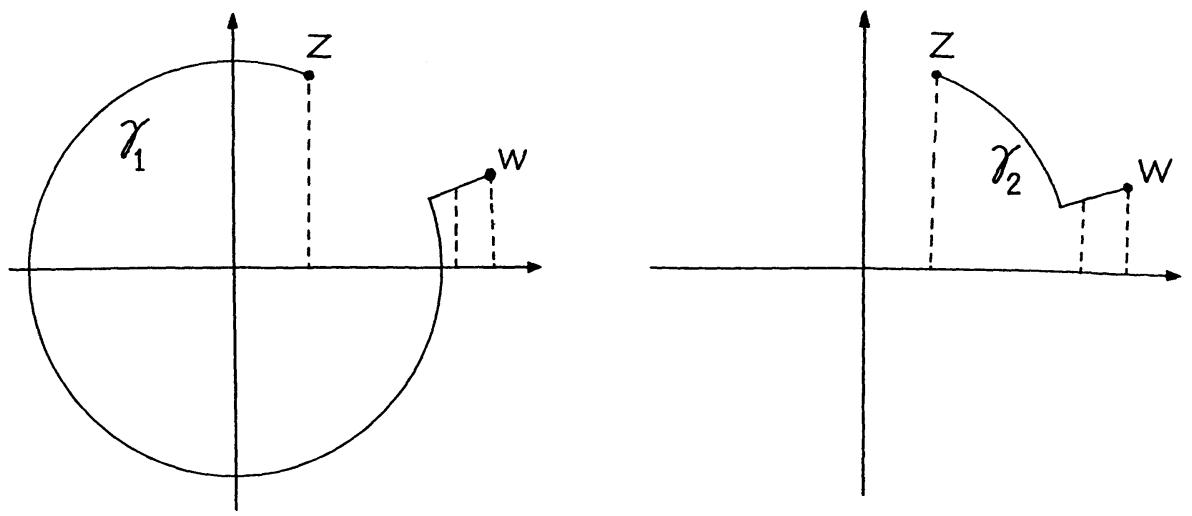

Fig. 3. The contours $\gamma_{1}$ and $\gamma_{2}$ in (2.12)

Taking into account all the phases, we obtain

$$
\begin{aligned}
& V_{(21) L+(10))}^{L}(z) V_{(211 L}^{L+(10)}(w)=i^{2 l+1} x^{\prime-1 / 4-l^{\prime} / 2}\left(x^{\prime 1 / 2} I_{1}+x^{\prime-1 / 2} I_{2}\right), \\
& V_{(21) L-(10))}^{L}(z) V_{(211 L}^{L-(10)}(w)=i^{2 l+1} x^{\prime-1 / 4}\left(x^{\prime\left(1-l^{\prime}\right) / 2} I_{1}+x^{\prime\left(l^{\prime}-1\right) / 2} I_{2}\right), \\
& V_{(21) L+(10)}^{L}(w) V_{(21) L}^{L+(10)}(z)=i^{2 l+1}\left(x^{\prime-l^{\prime} / 2} I_{1}+x^{\prime l^{\prime} / 2} I_{2}\right), \\
& V_{(21) L-(10)}^{L}(w) V_{(21) L}^{L-(10)}(z)=i^{2 l+1} x^{\prime} l^{\prime} / 2\left(I_{1}+I_{2}\right),
\end{aligned}
$$

where we have used the notation $x^{\prime}=\exp \left(2 \pi i \alpha_{-}^{2}\right)$. Eliminating $I_{1}$ and $I_{2}$ from the above equations gives the braid matrix

$$
\begin{aligned}
& R(L,(2,1),(2,1), L)_{L \pm(10), L \pm(10)}=\mp x^{\prime-1 / 4 \pm l^{\prime} / 2} \frac{x^{x^{1 / 2}}-x^{\prime-1 / 2}}{x^{\prime l^{\prime} / 2}-x^{\prime-l^{\prime} / 2}}, \\
& R(L,(2,1),(2,1), L)_{L \pm(10), L \mp(10)}=x^{\prime-1 / 4} \frac{x^{\prime\left(l^{\prime} \pm 1\right) / 2}-x^{\prime-\left(l^{\prime} \pm 1\right) / 2}}{x^{\prime l^{\prime} / 2}-x^{\prime-l^{\prime} / 2}} .
\end{aligned}
$$

The same expression without primes holds for the fields $(1,2)$ with $x=\exp \left(2 \pi i \alpha_{+}^{2}\right)$. The same analysis may be carried out in the third case, $L^{\prime}=L-(2,0)$, where one has two contours of integration, to be split into parts as above. The simple result is

$$
R(L-(2,0),(2,1),(2,1), L)_{L-(1,0), L-(1,0)}=e^{\pi i \alpha_{-}^{2} / 2},
$$

and similarly for $(21) \rightarrow(12)$. We are left with the computation of the braiding of $(2,1)$-fields with $(1,2)$-fields. Because of the identity $(2.4)$ and

$$
\oint_{w} V_{\alpha_{+}}(z) V_{\alpha_{21}}(w) d z=\oint_{w} V_{\alpha_{-}}(z) V_{\alpha_{12}}(w) d z=0,
$$

due to the regularity of the operator product expansion, we get no contributions from screening operators and we are left with the phase $\exp \left(\pi i 2 \alpha_{21} \alpha_{12}\right)=i^{-1}$ :

$$
R(A,(2,1),(1,2), C)_{B D}=R(A,(1,2),(2,1), C)_{B D}=i^{-1} .
$$

Let us summarize what we have computed. Let $x^{\prime}=\exp \left(2 \pi i \alpha_{-}^{2}\right), x=\exp \left(2 \pi i \alpha_{+}^{2}\right)$, and, for integers $l^{\prime}, l$, 


$$
[l]=x^{l / 2}-x^{-l / 2}, \quad\left[l^{\prime}\right]^{\prime}=x^{\prime l^{\prime} / 2}-x^{\prime-l^{\prime} / 2} .
$$

Then the braid matrices of screened vertex operators have the almost factorized form

$$
\begin{aligned}
R(A, & M, N, C)_{B D} \\
= & i^{-\left(m^{\prime}-1\right)(n-1)-\left(n^{\prime}-1\right)(m-1)}(-1)^{1 / 2(a-b+c-d)\left(n^{\prime}+m^{\prime}\right)+1 / 2\left(a^{\prime}-b^{\prime}+c^{\prime}-d^{\prime}\right)(n+m)} \\
& \cdot r^{\prime}\left(a^{\prime}, m^{\prime}, n^{\prime}, c^{\prime}\right)_{b^{\prime} d^{\prime}} r(a, m, n, c)_{b d},
\end{aligned}
$$

where the nonvanishing matrix elements of the "elementary" $r$-matrices are:

$$
\begin{aligned}
r(a, 1, n, c)_{a c} & =r(a, m, 1, c)_{c a}=1, \\
r(l \pm 2,2,2, l)_{l \pm 1, l \pm 1} & =x^{1 / 4}, \\
r(l, 2,2, l)_{l \pm 1, l \pm 1} & =\mp x^{-1 / 4 \mp l / 2 \frac{[1]}{[l]}} \\
r(l, 2,2, l)_{l \pm 1, l \mp 1} & =x^{-1 / 4} \frac{[l \pm 1]}{[l]},
\end{aligned}
$$

and the other $r$-matrices are given by the recursive relation

$$
\begin{aligned}
r(a, m+1, n, c)_{b d} & =\sum_{d_{1} \geqq 1} r\left(a, 2, n, d_{1}\right)_{a_{1} d} r\left(a_{1}, m, n, c\right)_{b d_{1}}, \\
r(a, m, n+1, c)_{b d} & =\sum_{d_{1} \geqq 1} r\left(a, m, 2, c_{1}\right)_{b d_{1}} r\left(d_{1}, m, n, c\right)_{c_{1} d},
\end{aligned}
$$

for any choice of $a_{1}$ and $c_{1}$ compatible with the fusion rules. The $r^{\prime}$ matrices are given by the same formulae with the replacement $x \rightarrow x^{\prime},[] \rightarrow[]^{\prime}$.

The reader may be worried that, although we have restricted ourselves to representation lables $\left(l^{\prime}, l\right)$ such that $l^{\prime}, l$ are positive, we may generate other representations by braiding. Indeed, the matrix element $r(1221)_{20}$ does not vanish. The point is that the operators $V_{(12)\left(l^{\prime} 1\right)}^{\left(l^{\prime}\right)}$ and $V_{(21)(1 l)}^{(0 l)}$ which are generated this way vanish identically on the space of BRST states, as shown in Appendix A.

By construction, the $r$-matrices have the symmetry

$$
r(a, m, n, c \mid x)_{b d}=r(c, n, m, a \mid x)_{b d} .
$$

It is easy to see inductively using (2.20) and (2.21) that as a matrix

$$
r(a, m, n, c \mid x)^{-1}=r\left(a, n, m, c \mid x^{-1}\right) .
$$

The recursion relations can also be written as

$$
\begin{aligned}
& r(a, m+1, n, c)_{b d}=\sum_{d_{1} \geqq 1} r\left(a, m, n, d_{1}\right)_{a_{1} d} r\left(a_{1}, 2, n, c\right)_{b d_{1}}, \\
& r(a, m, n+1, c)_{b d}=\sum_{d_{1} \geqq 1} r\left(a, m, n, c_{1}\right)_{b d_{1}} r\left(d_{1}, m, 2, c\right)_{c_{1} d} .
\end{aligned}
$$

We see that these matrices coincide up to irrelevant constants with the Boltzmann weights of critical SOS models defined in [13] in a special limit of the spectral parameter. The exact correspondence is the following. The Boltzmann weights in [13] depend on half periods $K, i K^{\prime}$, parameters $\xi, \lambda$, and the spectral parameter $u$. 
We have

$$
\begin{aligned}
r(b m n d \mid x)_{c a}= & x^{1 / 4(m-1)(m-n-1)}([m-1][m-2] \cdots[1])^{-1} \\
& \cdot \lim _{u \rightarrow-i \infty} \lim _{K^{\prime} \rightarrow \infty} x^{1 / 2 i u(m-1)(n-1)} W_{n-1, m-1}\left(a, b, c, d \mid u ; K, K^{\prime}, \xi=0, \lambda\right),
\end{aligned}
$$

where $x=\exp 2 \pi i(\lambda / 2 K)$ and we assume here that $\operatorname{Re}(\lambda / 2 K)>0 . \operatorname{If} \operatorname{Re}(\lambda / 2 K)<0$ the same result can be obtained in the limit $u \rightarrow+i \infty$.

\section{Crossing Symmetry and Quantum Groups}

We have seen in the preceding section that the braid matrices of Dotsenko-Fateev models are given by critical SOS weights in the limit where the spectral parameter goes to $-i \infty$. These weights are proportional to the (quantum) $6 j$-coefficients of the quantum group $U_{q}(S U(2))[16,17]$. We will see that an importat property of quantum groups, called crossing symmetry [16], implies the crossing symmetry of the conformal invariant theory, which is the essential consistency condition on the plane.

Rather than using the general theory of quantum groups, it is easier, in this simple $S U(2)$ case, to rederive the quantum group crossing symmetry property directly in the SOS language, using our explicit expressions. We find that the most illuminating approach to this property comes from the application of quantum groups to knot theory, which we shortly review, following [16,17]. From the set of $r$-matrices one constructs link invariants in the following way: Given a link in $\mathbb{R}^{3}$, we project it into a plane with a preferred direction, which we call horizontal. To every connected component of the link and to every connected component of the complement of its projection one assigns a positive integer label. The unbounded component is assigned the label one. Figure 4 shows an example. One then splits the link into elementary constituents and assigns to them functions of $x$ :
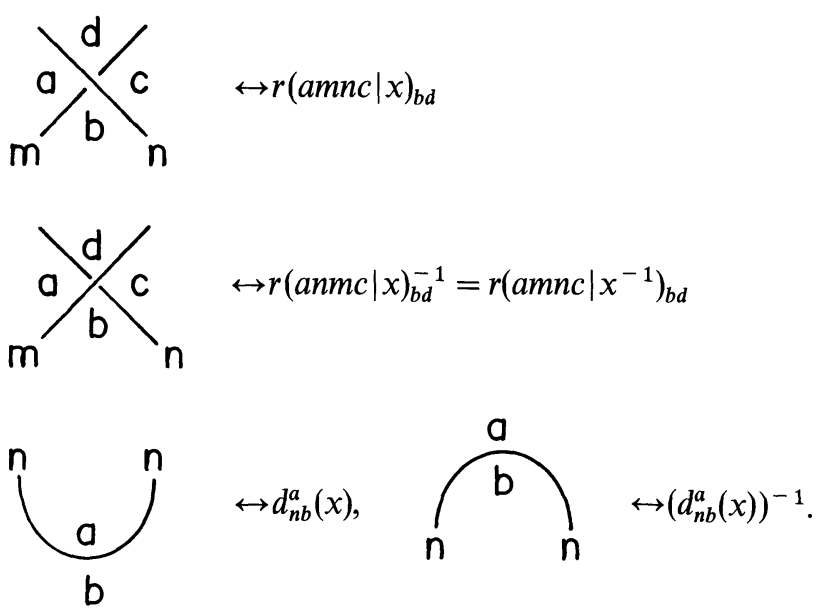


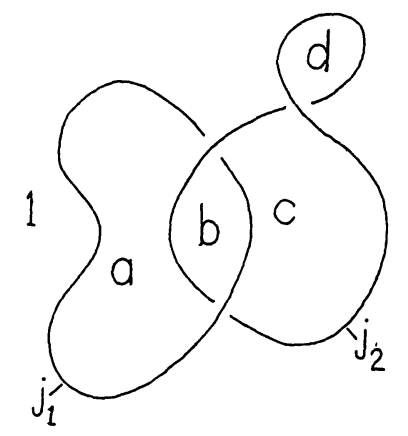

Fig. 4. Example of a projected link with assignments

The first two constituents are called positive and negative crossings, respectively. The last two constituents correspond to the points of the projected links with horizontal tangent. One then sums the product of these functions of $x$ over all labels assigned to the regions bounded by the links and multiplies the result by the factor

$$
\prod_{\alpha}\left[(-1)^{j_{\alpha}+1} x^{1 / 4\left(j_{\alpha}^{2}-1\right)}\right]^{N-(\alpha)-N_{+}(\alpha)},
$$

where the product is over all connected components $\alpha$, with label $j_{\alpha}$, of the given link, and $N_{+}(\alpha)\left(N_{-}(\alpha)\right)$ is the number of positive (negative) self-crossings of the component $\alpha$. The point is that the resulting quantity is a link invariant. This can be shown to follow from a finite set of relations involving $r$ and $d$; one of which is the celebrated star-triangle equation. Another relation, called crossing symmetry, determines $d_{j k}^{i}$ in terms of $r$-matrices. It is represented in Fig. 5 and reads:

$$
d_{m c}^{b}(x) r(b m n d \mid x)_{c a}=r\left(c n m a \mid x^{-1}\right)_{d b} d_{m d}^{a}(x) .
$$

We show in an Appendix that the solution for $d_{j k}^{i}(x)$ is

$$
d_{j k}^{i}(x)= \begin{cases}(-1)^{1 / 2(k+j-i-1)}[i] & \prod_{l=1 / 2(i+j-k+1)}^{j-1}[l] \prod_{l=1 / 2(k+j-i+1)}^{j-1}[l] \prod_{l=1 / 2(i+k-j+1)}^{1 / 2(i+k+j-1)}[l]^{-1}, \\ 0, & \text { if } \quad|i-j|+1 \leqq k \leqq i+j-1, \quad k+j-i-1 \equiv 0 \bmod 2,\end{cases}
$$

with the convention that the product over the empty set is one. In quantum group language, property (3.2) expresses a relation between the antipode and the $R$-matrix (see [16]), but this will not be important to us in this simple example. For completeness, let us quote the remaining relation, which corresponds to "stretching a loop" in the projected link,

$$
\sum_{b} r(a, j, j, b \mid x)_{c c}^{ \pm 1} d_{j c}^{b}(x)\left(d_{j b}^{c}(x)\right)^{-1}=(-1)^{j-1} x^{ \pm\left(j^{2}-1\right) / 4} .
$$

This relation shows the role of the extra factor in the computation of the invariant. To make contact with minimal models, let us write the relation (3.2) in terms of full braid matrices: 


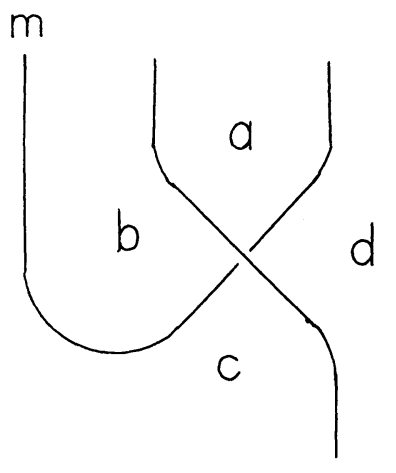

n

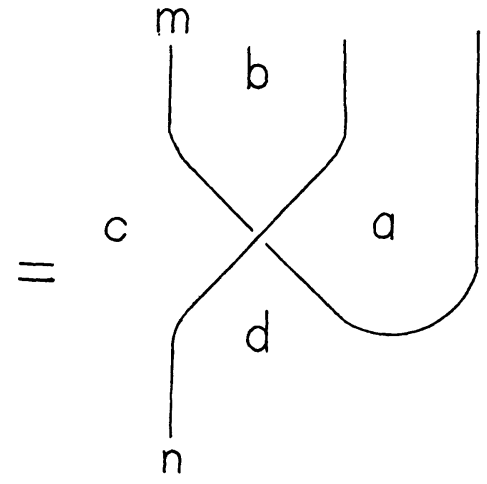

Fig. 5. Crossing symmetry relation

$$
\begin{aligned}
\tilde{D}_{M C}^{B}\left(x^{\prime}, x\right) R\left(B M N D \mid x^{\prime}, x\right)_{C A} & =R\left(C N M A \mid x^{\prime-1}, x^{-1}\right)_{D B} \tilde{D}_{M D}^{A}\left(x^{\prime}, x\right), \\
\tilde{D}_{M C}^{B}\left(x^{\prime}, x\right) & =d_{m^{\prime} c^{\prime}}^{b^{\prime}}\left(x^{\prime}\right) d_{m c}^{b}(x) .
\end{aligned}
$$

One iteration of this relation gives, using the symmetry (2.22), and $\widetilde{D}_{M C}^{B}\left(x^{\prime}, x\right)=$ $\widetilde{D}_{M C}^{B}\left(x^{\prime-1}, x^{-1}\right)$,

$$
\begin{aligned}
& R\left(B M N D \mid x^{\prime}, x\right)_{C A} \widetilde{D}_{M C}^{B}\left(x^{\prime}, x\right) \widetilde{D}_{N D}^{C}\left(x^{\prime}, x\right) \\
& \quad=R\left(B N M D \mid x^{\prime}, x\right)_{A C} \tilde{D}_{N A}^{B}\left(x^{\prime}, x\right) \widetilde{D}_{M D}^{A}\left(x^{\prime}, x\right) .
\end{aligned}
$$

But this is the condition of crossing symmetry (or locality) of (left-right symmetric) conformal field theory, relating $R$-matrices to the $D$-coefficients of the decomposition of primary fields into chiral components. It expresses the fact that Euclidean Green functions are single valued and symmetric under permutations. We have written $\widetilde{D}$ instead of $D$, in (3.5), since the normalization is not yet fixed. Indeed, if $\tilde{D}_{J K}^{I}$ is a solution of (3.5), also $\lambda_{I} \sigma_{J} \lambda_{K}^{-1} \widetilde{D}_{J K}^{I}$ is a solution. This freedom corresponds to rescaling the fields and rescaling highest weight vectors. Conventionally, the normalization is fixed by the requirement that the two-point function be normalized as

$$
\left\langle\Phi_{I}(z, \bar{z}) \Phi_{J}(w, \bar{w})\right\rangle=\frac{\delta_{I J}}{|z-w|^{4 h}},
$$

and the highest weight states be normalized as

$$
v_{I} \otimes v_{I}=\Phi_{I}(0) \Omega,
$$

where $\Omega=v_{(1,1)} \otimes v_{(1,1)}$ is the vacuum. These requirements relate the $D$-coefficients to the normalization constants

$$
N_{J K}^{I}=\left\langle v_{I}^{*}, V_{J K}^{I}(1) v_{K}\right\rangle
$$

The normalization conditions (3.7), (3.8) become

$$
D_{J J}^{(11)}\left(N_{J J}^{(11)}\right)^{2}=D_{J(11)}^{J}\left(N_{J(11)}^{J}\right)^{2}=1 .
$$


With these conventions, the $D$ coefficients read

$$
\begin{aligned}
D_{J K}^{I}= & N_{I I}^{(11)}\left(N_{J J}^{(11)}\right)^{-1}\left(N_{K K}^{(11)}\right)^{-1} \Delta_{j^{\prime} k^{\prime}}^{i^{\prime}}\left(x^{\prime}\right) \Delta_{j k}^{i}(x), \\
\Delta_{j k}^{i}(x)= & (-1)^{1 / 2(k+j-i-1)}\left(\frac{[i][j][k]}{[1]}\right)^{1 / 2} \\
& \cdot \prod_{l=1 / 2(i+j-k+1)}^{j-1}[l] \prod_{l=1 / 2(k+j-i+1)}^{j-1}[l]\left(\prod_{l=1 / 2(i+k-j+1)}^{1 / 2(i+k+j-1)}[l]\right)^{-1}
\end{aligned}
$$

The structure constants of the operator product expression are related to the $D$-coefficients by the relation

$$
C_{J K}^{I}=D_{J K}^{I}\left(N_{J K}^{I}\right)^{2} .
$$

The normalization constants were computed in [24] in terms of Dotsenko-Fateev integrals [20]. We give them here for completeness:

$$
\begin{aligned}
N_{N M}^{L}= & (-1)^{n^{\prime} r+n r^{\prime}} \alpha_{+}^{4 r^{\prime} r} \prod_{j^{\prime}=1}^{r^{\prime}} \frac{\left[m^{\prime}-j^{\prime}\right]^{\prime}\left[j^{\prime}\right]^{\prime}}{\sqrt{-1}[1]^{\prime}} \prod_{j=1}^{r} \frac{[m-j][j]}{\sqrt{-1}[1]} \\
& \prod_{j^{\prime}=1}^{r^{\prime}} \frac{\Gamma\left(j^{\prime} \alpha_{-}^{2}\right) \Gamma\left(m+\left(j^{\prime}-m^{\prime}\right) \alpha_{-}^{2}\right) \Gamma\left(n+\left(j^{\prime}-n^{\prime}\right) \alpha_{-}^{2}\right)}{\Gamma\left(\alpha_{-}^{2}\right) \Gamma\left(m+n-2 r+\left(r^{\prime}-m^{\prime}-n^{\prime}+j^{\prime}\right) \alpha_{-}^{2}\right)} \\
& \prod_{j=1}^{r} \frac{\Gamma\left(j \alpha_{+}^{2}-r\right) \Gamma\left(m^{\prime}-r^{\prime}+(j-m) \alpha_{+}^{2}\right) \Gamma\left(n^{\prime}-r^{\prime}+(j-n) \alpha_{+}^{2}\right)}{\Gamma\left(\alpha_{+}^{2}\right) \Gamma\left(m^{\prime}-r^{\prime}+n^{\prime}+(r-m-n+j) \alpha_{+}^{2}\right)},
\end{aligned}
$$

where $l^{(\prime)}=n^{(\prime)}+m^{(\prime)}-2 r^{(\prime)}-1$. In spite of its appearance, this expression is actually symmetric in $\alpha_{+}^{2} \leftrightarrow \alpha_{-}^{2}, l^{\prime} \leftrightarrow l, n^{\prime} \leftrightarrow n, m^{\prime} \leftrightarrow m$ [20].

\section{Minimal Models}

So far, we have considered the Dotsenko-Fateev models, where the value of the central charge is a generic complex number. These models are non-minimal (they involve infinitely many primary fields) and non-unitary. The Virasoro representations are simply degenerate, i.e., there exists only one singular vector (besides the highest weight vector) in each Verma module. Subalgebras of these models are believed to describe the critical behavior of $q$-state Potts models and $O(N)$ models with $q, N$ non-integer [19]. As we have seen, the corresponding braid matrices are related to quantum group representations with generic quantum group parameter.

The interesting limit is when the parameter $\alpha_{-}^{2}$, related to the central charge by $c=13-6 \alpha_{-}^{2}-6 \alpha_{-}^{-2}$, approaches a positive rational value. In this limit, the discrete series of minimal models with $c<1$ appears.

Some peculiar phenomena arise at these rational points: the Verma modules of degenerate representations have infinitely many singular vectors, and the BRST cohomology of the corresponding Fock spaces becomes more complicated [24]. Chiral primary fields exist only for a more restricted set of representation triples, and one can select a finite number of primary fields that form, together with their descendants, a closed operator algebra. On the quantum group side, this limit corresponds to quantum group parameters which are roots of unity. In this limit, some matrix elements of the braid matrices diverge. 
In this section we show that divergent matrix elements to not appear in the braiding of screened vertex operators which are nonvanishing on BRST states. It is sufficient to check this for the elementary fields $V_{(12) M}^{L}, V_{(21) M}^{L}$, since all other fields are obtained by fusion. Indeed, although the rigorous theory of the meromorphic continuation and fusion of screened vertex operators for general $\alpha_{-}^{2}$ is not yet complete, it is clear that equations (2.3) continue to be valid for $\alpha_{-}^{2}>0$, provided a divergent contribution in the operator product is subtracted before taking the limit.

In minimal models, the irreducible representation spaces of the Virasoro algebra have highest weights $h_{n^{\prime} n}$ such that $\left(n^{\prime}, n\right) \in K=\left\{1 \leqq n^{\prime} \leqq p^{\prime}-1,1 \leqq n \leqq p-1\right\}$, where $\alpha_{-}^{2}=p / p^{\prime}$. By the identification with the BRST cohomology of corresponding Fock spaces [24], the chiral primary fields are constructed by fusion of the fields $V_{(21) M}^{L}$, $V_{(12) M}^{L}$, so that $L$ and $M$ are in $K$. Consistency requires that braiding of fields in this family does not generate other fields. We see from the explicit formulae (2.19), (2.20) that the braid matrices of this family of fields do not contain divergent matrix element. On the other hand, we also see that braiding two of these fields we generate fields $V_{(21) M}^{L}(z), V_{(12) M}^{L}(z)$ with $M \in K, L \in \bar{K}=\left\{0 \leqq n^{\prime} \leqq p^{\prime}, 0 \leqq n \leqq p\right\}$ or $M \in \bar{K}$, $L \in K$. The point is that screened vertex operators are BRST invariant and therefore map BRST states of BRST states. Now, for $L \in \bar{K}-K$, the boundary of the Kac table, the BRST cohomology of the Fock space $F_{L}$ vanishes and BRST invariant operators mapping from or to $F_{L}$ vanish identically on BRST states.

Thus general $R$ matrices for minimal models are given by the formulae (2.19)-(2.21), with the summation in (2.21) restricted to $d_{1} \leqq p-1$. Moreover one has to take into account that screened vertex operators $V_{N M}^{L}$ vanish identically on BRST states unless the fusion rules $n^{(\prime)}+m^{(\prime)}+l^{(\prime)} \equiv 1(\bmod 2)$ and $\left|n^{(\prime)}-n^{(\prime)}\right|+1 \leqq$ $l^{\left({ }^{\prime}\right)} \leqq \min \left(n^{\left({ }^{\prime}\right)}+m^{\left({ }^{\prime}\right)}-1,2 p^{\left({ }^{\prime}\right)}-n^{\left({ }^{\prime}\right)}-m^{\left({ }^{\prime}\right)}-1\right)$ are fulfilled. Let us call $F(L, N, M)$ this fusion rule and $F_{0}(L, N, M)$ the $S U(2)$-fusion rule (2.6). For generic $\alpha_{-}^{2}$, the $R$ matrix elements (2.19) are by construction nonvanishing if and only if the fusion rules $F_{0}(A, M, B), F_{0}(B, N, C), F_{0}(A, N, D), F_{0}(D, M, C)$ are fulfilled. If $\alpha_{-}^{2}=p / p^{\prime}$, the $R$-matrix elements $R(A M N C)_{B D}$ which arise from the braiding of chiral fields obeying $F$-fusion rules are those for which $F(A, M, B), F(B, N, C), F(A, N, D)$, $F(D, M, C)$ are valid. We can set all other matrix elements to zero. The following important property follows by inspection: Suppose that, for $\alpha_{-}^{2}=p / p^{\prime}$, the left-hand side of (2.10) is nonvanishing, i.e. the corresponding $F$-fusion rules are fulfilled. Then the set of labels $A_{1}$ and $D_{1}$ so that the $F$-fusion rules are fulfilled for both $R$ matrices on the right-hand side coincides with the set of labels for which the $F_{0}$-fusion rules are filfilled. A corollary of this property is that although some $R$-matrix elements are set to zero at $\alpha_{-}^{2}=p / p^{\prime}$, the nonvanishing elements are analytic in $\alpha_{-}^{2}$ at the rational points. In particular, the explicit expressions of [13] can be taken over.

The same reasoning is valid for the structure constants. In the recursive definition (B.5)

$$
\begin{aligned}
\tilde{D}_{J+(10), K}^{I} & =\sum_{I_{1}} \tilde{D}_{(21) I_{1}}^{I} \tilde{D}_{J K}^{I_{1}} \frac{\left[j^{\prime}\right]^{\prime}}{[1]^{\prime}}, \\
\tilde{D}_{J+(01), K}^{I} & =\sum_{I_{1}} \tilde{D}_{(12) I_{1}}^{I} \tilde{D}_{J K}^{I_{1}} \frac{[j]}{[1]},
\end{aligned}
$$


if the fusion rule is fulfilled for the labels on the left-hand side, then the value of $I_{1}$ for which $F_{0}$ holds for both $\tilde{D}$ 's on the right-hand side are the same as the values for which $F$ holds. In particular, the explicit formulae (3.10), (3.11) for the structure constants are valid for minimal models provided $F(I, J, K)$ is fulfilled. If $F(I, J, K)$ is not fulfilled, $D_{J K}^{I}=C_{J K}^{I}=0$.

Summarizing, we have shown that, for $\alpha_{-}^{2}=p / p^{\prime}$, the braid matrix elements $R(A M N C)_{B D}$ and the structure constants $D_{J K}^{I}$ vanish unless the fusion rules $F(A, M, B), F(B, N, C), F(A, N, D), F(D, M, C)$ and $F(I, J, K)$ are fulfilled. If they do not vanish, they are given by the same expressions (2.19)-(2.21) and (3.10), as for generic $\alpha_{-}^{2}$.

\section{Appendix A}

We describe here the BRST cohomology of Fock spaces needed in this paper. The computations are based on the results of Feigin and Fuchs [28] on the composition series of Fock modules. For details see [24]. Let us first recall the definitions. The Fock space $F_{m^{\prime} m}$ is a module over the infinite dimensional Heisenberg algebra $\left[a_{n}, a_{m}\right]=2 n \delta_{n,-m}$. It is generated by a vacuum vector $v_{m^{\prime} m}$ such that $a_{n} v_{m^{\prime} m}=0$, $n>0$ and $a_{0} v_{m^{\prime} m}=2 \alpha_{m^{\prime} m^{\prime} m} v_{m^{\prime} m}$, with $\alpha_{m^{\prime} m}=\frac{1}{2}\left(1-m^{\prime}\right) \alpha_{-}-\frac{1}{2}(1-m) \alpha_{-}^{-1}$. The definition

$$
\begin{aligned}
& L_{n}=\frac{1}{4} \sum_{k \in Z} a_{n-k} a_{k}-\alpha_{0}(n+1) a_{n}, \quad n \neq 0, \\
& L_{0}=\frac{1}{2} \sum_{k=1}^{\infty} \alpha_{-k} \alpha_{k}+\frac{1}{4} a_{0}^{2}-\alpha_{0} a_{0},
\end{aligned}
$$

where $2 \alpha_{0}=\alpha_{-}-\alpha_{-}^{-1}$ gives $F_{m^{\prime} m}$ the structure of a graded (by $L_{0}$ ) Virasoro module with highest weight $h_{m^{\prime} m}=\frac{1}{4}\left(m^{\prime 2}-1\right) \alpha_{-}^{2}-\frac{1}{2}\left(m^{\prime} m-1\right)+\frac{1}{4}\left(m^{2}-1\right) \alpha_{-}^{-2}$ and central charge $c=13-6 \alpha_{-}^{2}-6 \alpha_{-}^{-2}$. Let $V_{h_{m^{\prime} m}, c}$ be the corresponding Verma module with highest weight vector $w_{m^{\prime} m}$, and $\phi_{m^{\prime} m}$ be the canonical homomorphism of graded Virasoro modules mapping $w_{m^{\prime} m}$ to $v_{m^{\prime} m}$.

Let us first consider the case where $\alpha_{-}^{2} \in \mathbb{C} \backslash \mathbb{Q}$. By the Kac determinant formula, if $m^{\prime} m \leqq 0, V_{h_{m^{\prime} m}, c}$ is irreducible, so $\operatorname{Ker} \phi_{m^{\prime} m}=0$. Thus, since the dimensions of the subspaces of fixed degree of $V_{h_{m^{\prime} m}, c}$ and $F_{m^{\prime} m}$ coincide, $\phi_{m^{\prime} m}$ is an isomorphism and $F_{m^{\prime} m}$ is also irreducible. If $m^{\prime}<0$ and $m<0, V_{h_{m^{\prime} m}, c}$ contains the irreducible submodule generated by a singular vector $\chi$ of weight $h_{m^{\prime} m}+m^{\prime} m=h_{m^{\prime},-m}$. The BRST operator $Q_{-m}: F_{m^{\prime},-m} \rightarrow F_{m^{\prime} m}$ is an injective homomorphism of graded Virasoro modules. This can be shown by explicitly checking the sufficient condition $Q_{-m} v_{m^{\prime},-m} \neq 0$ (see the Appendix in [24]). The image of $v_{m^{\prime},-m}$ is a non-zero singular vector of $F_{m^{\prime} m}$ and generates an irreducible submodule. Now, either $\phi_{m^{\prime} m}$ is an isomorphism or it is not [A more careful analysis shows that the first possibility is realized]. If it is, $Q_{-m} v_{m^{\prime},-m}$ must be proportional to $\phi_{m^{\prime} m} \chi$, and the irreducible factor module $\mathscr{H}_{m^{\prime} m}=V_{h_{m^{\prime} m}, c} / V_{h_{m^{\prime} m}+m^{\prime} m, c}$ is isomorphic to $F_{m^{\prime}, m} / Q_{-m} F_{m^{\prime},-m}$. If $\phi_{m^{\prime} m}$ is not an isomorphism, $\phi_{m^{\prime} m} \chi$ vanishes, $Q_{-m^{\prime}} v_{m^{\prime},-m} \notin \operatorname{Im} \phi_{m^{\prime} m}$, and $\phi_{m^{\prime} m}$ projects to an injective homomorphism from $\mathscr{H}_{m^{\prime} m}$ to $F_{m^{\prime}, m} / Q_{-m} F_{m^{\prime},-m}$. Counting the dimensions shows this homomorphism is also surjective, and we have the isomorphism of graded Virasoro modules

$$
\mathscr{H}_{m^{\prime} m} \cong F_{m^{\prime}, m} / Q_{-m} F_{m^{\prime},-m} .
$$


The latter space is called the space of BRST states. The case $m^{\prime}>0, m>0$ is treated using duality. The dual (or contragradient) module $F_{m^{\prime} m}^{*}$ with Virasoro structure defined by ${ }^{t} L_{n}=L_{-n}$ is isomorphic as a graded Virasoro module to $F_{-m^{\prime},-m}$. Moreover, with this identification, one has ${ }^{t} Q_{m}=Q_{m}$. In this case the isomorphism is

$$
\mathscr{H}_{m^{\prime} m} \cong \operatorname{Ker}\left(Q_{m}: F_{m^{\prime} m} \rightarrow F_{m^{\prime},-m}\right) \text {. }
$$

Let us next consider the role of the BRST operator if $m^{\prime}$ or $m$ are zero. Although this case was excluded in [24], the computation of the Appendix to [24] shows that $Q_{m}: F_{0, m} \rightarrow F_{0,-m}$ is injective and, since $h_{0, m}=h_{0,-m}$ it is an isomorphism. For later purposes let us extended the definition of $Q_{m}$ to $m=0$ by setting $Q_{0}=1$ : $F_{m^{\prime} 0} \rightarrow F_{m^{\prime} 0}$.

The rational case $\alpha_{-}^{2}=p / p^{\prime}$ is more complicated and was analyzed in [24]. One consider the BRST operators

$$
F_{m^{\prime}-p^{\prime}, p-m} \stackrel{Q_{p-m}}{\longrightarrow} F_{m^{\prime} m} \stackrel{Q_{m}}{\longrightarrow} F_{m^{\prime},-m}
$$

for $1 \leqq m^{\prime} \leqq p^{\prime}-1,1 \leqq m \leqq p-1$. Note that $\alpha_{m^{\prime}-p^{\prime}, m-p}=\alpha_{m^{\prime} m}$. It was shown in [24] that $Q_{m} Q_{p-m}=0$ and that the irreducible foactor module $\mathscr{H}_{m^{\prime} m}$, which is the quotient of the corresponding Verma module by its maximal proper submodule, is isomorphic to the space of BRST states

$$
\mathscr{H}_{m^{\prime} m} \simeq \operatorname{Ker} Q_{m} / \operatorname{Im} Q_{p-m}, \quad 1 \leqq m^{(')} \leqq p^{(')}-1 .
$$

This ends our discussion of the BRST cohomology of Fock spaces. We turn to screened vertex operators.

Let us abbreviate $\left(m^{\prime}, m\right)$ by $M$ and $\left(m^{\prime},-m\right)$ by $\hat{M}$. Screened vertex operators are BRST invariant:

$$
Q_{l} V_{N M}^{L}(z)=V_{N \hat{M}}^{\hat{L}}(z) Q_{m}, \quad l, m \geqq 0 .
$$

Actually there is a phase in this formula which can however be absorbed in a redefinition of the BRST operator. If $m$ or $l$ vanish in (A.8) the formula continues to be valid provided one identifies $Q_{0}$ with the identity operator. In this paper we are concerned with operators $V_{N M}^{L}$ with $l^{\prime}, l, m^{\prime}, m \geqq 0$ and $n^{\prime}, n \geqq 1$, for generic $\alpha_{-}^{2}$ and with $0 \leqq l^{\left({ }^{\prime}\right)}, m^{\left({ }^{\prime \prime}\right)} \leqq p^{\left({ }^{\prime}\right)}$ and $1 \leqq n^{(\prime)} \leqq p^{(\prime)}-1$ for $\alpha_{-}^{2}=p / p^{\prime}$. We see that these operators map BRST states to BRST states and

$$
V_{N M}^{L}(z) \equiv 0
$$

on BRST states if $\alpha_{-}^{2}$ is generic and $l^{\prime}, l, m^{\prime}$ or $m$ vanish. If $\alpha_{-}^{2}=p / p^{\prime}$ a complete analysis would require computing the BRST cohomology in the case where $l^{(\prime)}=0 \bmod p^{(\prime)}$; this case was not analyzed in [24], and is a little tricky. Fortunately, however, to establish that some screened vertex operators vanish, it is not necessary to know the whole structure. Consider the operator

$$
V_{\left(n^{\prime} n\right)\left(m^{\prime} m\right)}^{\left(l^{\prime}\right)}(z), 1 \leqq l^{\prime}, n^{\prime}, m^{\prime} \leqq p^{\prime}-1, \quad 1 \leqq n, \quad m \leqq p-1 .
$$

By (A.8), it maps the kernel of $Q_{m}$ to the kernel of $Q_{0}=1$. Thus it vanishes on BRST states. Similarly, the operator

$$
V_{\left(n^{\prime} m\right)\left(m^{\prime} m\right)}^{\left(l^{\prime}\right)}(z), 1 \leqq l^{\prime}, n^{\prime}, m^{\prime} \leqq p^{\prime}-1, \quad 1 \leqq n, \quad m \leqq p-1
$$


maps the image of $Q_{p-m}$ to the image of $Q_{0}=1$, and vanishes on the cohomology. We see that the operators

$$
V_{N M}^{L}(w) V_{R S}^{M}(z)
$$

with $L, N, R, S \in K$ and $M=\left(m^{\prime}, p\right)$ or $\left(m^{\prime}, 0\right)$, that are generated by braiding, vanish on BRST states. By the fundamental symmetry $\alpha_{-}^{2} \leftrightarrow \alpha_{+}^{2},\left(n^{\prime}, n\right) \leftrightarrow\left(n, n^{\prime}\right)$, the same conclusion can be drawn if, in (A.10), $M=\left(p^{\prime}, m\right)$ or $(0, m)$.

\section{Appendix B}

This appendix contains the proof that (3.3) is a solution of (3.2). First we solve

$$
d_{2 c}^{b}(x) r(b 22 d \mid x)_{c a}=r\left(c 22 a \mid x^{-1}\right)_{d b} d_{2 d}^{a}(x) .
$$

The special case

$$
d_{2, l \pm 1}^{l \pm 2}(x) r(l \pm 2,2,2, l \mid x)_{l \pm 1, l \pm 1}=r\left(l \pm 1,2,2, l \pm 1 \mid x^{-1}\right)_{l, l \pm 2} d_{2, l}^{l \pm 1}(x)
$$

gives the recursion relations

$$
d_{2, l+1}^{l+2}(x)=\frac{[l]}{[l-1]} d_{2, l}^{l+1}(x), \quad d_{2, l}^{l-1}=\frac{[l-1]}{[l]} d_{2, l-1}^{l-2}
$$

which can be easily solved. The solution is checked to be consistent by inserting it back into (B.1). The unique solution of (B.1) normalized by $d_{21}^{2}=1$ is

$$
d_{2, l}^{l \pm 1}(x)= \pm \frac{[1]}{[l]}
$$

The other $d$-coefficients are given by fusion

$$
d_{j k}^{i}(x)=\sum_{i_{1}} d_{2 i_{1}}^{i}(x) d_{j-1, k}^{i_{1}}(x) \frac{[j-1]}{[1]} .
$$

The last factor enforces the normalization $d_{j 1}^{j}=1$.

The crossing relations (3.2) are verified inductively using the recursive definition of $r$-matrices and (A.5). We do here the most complicated induction step explicitly:

$$
\begin{aligned}
& r(a, m+1, n, c \mid x)_{b d} d_{m+1, b}^{a}(x) \\
& \quad=\sum_{d_{1} i} r\left(a, 2, n, d_{1} \mid x\right)_{a_{1} d} r\left(a_{1}, m, n, c \mid x\right)_{b d_{1}} d_{2 i}^{a}(x) d_{m b}^{i}(x) \frac{[m]}{[1]} \\
& \quad=\sum_{i} \sum_{d_{1}} r\left(a, 2, n, d_{1} \mid x\right)_{i d} r(i, m, n, c \mid x)_{b d_{1}} d_{2 i}^{a}(x) d_{m b}^{i}(x) \frac{[m]}{[1]} \\
& =\sum_{d_{1}} \sum_{i} r\left(i, n, 2, d \mid x^{-1}\right)_{d_{1} a} r\left(b, n, m, d_{1} \mid x^{-1}\right)_{c i} d_{2 d_{1}}^{d}(x) d_{m c}^{d_{1}}(x) \frac{[m]}{[1]} \\
& =\sum_{d_{1}} r\left(b, n, m+1, d \mid x^{-1}\right)_{c a} d_{2 d_{1}}^{d}(x) d_{m c}^{d_{1}}(x) \frac{[m]}{[1]} \\
& =r\left(b, n, m+1, d \mid x^{-1}\right)_{c a} d_{m+1, c}^{d}(x) .
\end{aligned}
$$


It is not difficult to prove by induction that (3.3) is the solution of the recursion relation (B.5).

Acknowledgement. G. F. wishes to thank D. Bernard for useful discussions on quantum groups.

\section{References}

1. Tsuchiya, A., Kanie, Y.: Vertex operators in the conformal field theory of $P_{1}$ and monodromy representations of the braid group. Lett. Math. Phys. 13, 303-312 (1987)

2. Kohno, T.: Linear representations of braid groups and classical Yang-Baxter equations. In: Artin's Braid Groups. Contemporary Mathematics (to appear)

3. Fröhlich, J.: Statistics of fields, the Yang-Baxter equation and the theory of knot and links. To appear in: Non-perturbative Quantum Field Theory. Cargèse lectures 1987, G. 't Hooft et al. (eds.). New York: Plenum Press

4. Rehren, K.-H.: Locality of conformal fields in two dimensions: Exchange algebra on the light cone. Commun. Math. Phys. 116, 675-685 (1988)

5. Rehren, K.-H., Schroer, B.: Einstein causality and Artin's braids. Berlin preprint FU-88-0439

6. Vafa, C.: Towards classification of conformal theories, Phys. Lett. B206, 421-426 (1988)

7. Verlinde, E.: Fusion rules and modular transformations in $2 d$ conformal field theory. Nucl. Phys. B300 [FS22], 360-376 (1988)

8. Moore, G., Seiberg, N.: Polynomial equations for rational conformal field theories. Phys. Lett. B212, 451-460 (1988); Naturality in conformal field theory. Nucl. Phys. B313, 16-40 (1989); Classical and quantum conformal field theory. IAS preprint IASSNS-88/39

9. Brustein, R., Yankielowicz, S., Zuber, J.-B.: Factorization and selection rules of operator product algebras in conformal field theory. Saclay/Tel Aviv preprint SPhT/88-086, TAUP-1647-88

10. Dijkgraaf, R., Verlinde, E.: Modular invariance and the fusion algebra. To appear in the Proceedings of the Annecy conference 1988

11. Felder, G., Fröhlich, J., Keller, G.: On the structure of unitary conformal field theories. Commun. Math. Phys. (in press)

12. Belavin, A., Polyakov, A. M., Zamolodchikov, A. B.: Infinite conformal symmetry in twodimensional quantum field theory. Nucl. Phys. B241, 333-380 (1984)

13. Date, E., Jimbo, M., Miwa, T., Okado, M.: Fusion of the eight vertex SOS model, Lett. Math. Phys. 12, 209-215 (1986); Erratum and Addendum. Lett. Math. Phys. 14, 97 (1987)

14. Jimbo, M.: A $q$-difference analogue of $U(\mathfrak{g})$ and the Yang-Baxter equation. Lett. Math. Phys. 10, 63-69 (1985); Quantum $R$ matrix for the generalized Toda system. Commun. Math. Phys. 102, 537-547 (1986)

15. Drinfeld, V. G.: Quantum groups. Proceedings of the International Congress of Mathematicians 1986, pp. 798-820

16. Reshetikhin, N. Yu.: Quantized universal enveloping algebras, the Yang-Baxter equation and invariants of links, I and II. LOMI preprints E-4-87, E-17-87

17. Kirillov, A. M., Reshetikhin, N. Yu.: Representations of the algebra $U_{q}(s l(2))$, q-orthogonal polynomials and invariants of links. LOMI preprint E-9-88

18. Christe, P.: Ph.D. Thesis, Bonn 1987

19. Dotsenko, V.S., Fateev, V. A.: Conformal algebra and multipoint correlation functions in $2 d$ statistical models. Nucl. Phys. B240 [FS12], 312-348 (1984)

20. Dotsenko, V.S., Fateev, V. A.: Four-point correlation functions and operator algebra in $2 d$ conformal invariant theories with central charge $\leqq 1$. Nucl. Phys. B251 [FS13], 691-734 (1985)

21. Jones, V. F. R.: Hecke algebra representations of braid groups and link polynomials. Ann. Math. 126, 335-388 (1987)

22. Witten, E.: Quantum field theory and the Jones polynomial. Commun. Math. Phys. 121, 351-399 (1989)

23. Friedan, D.: Qiu, Z., Shenker, S.: Conformal invariance, unitary and critical exponents in two dimensions. Phys. Rev. Lett. 52, 1575-1578 (1984)

24. Felder, G.: BRST approach to minimal models. Nucl. Phys. B317, 215-237 (1989) 
25. Dotsenko, V. S., Fateev, V. A.: Operator algebra of two-dimensional conformal theories with central charge $\leqq 1$, Phys. Lett. B154, 291-295 (1985)

26. Fateev, V. A., Lykyanov, S. L.: The models of two-dimensional conformal quantum field theory with $Z_{n}$ symmetry. Int. J. Mod. Phys. A3, 507-520 (1988)

27. Gervais, J.-L., Neveu, A.: Novel triangle relation and absence of tachyons in Liouville string field theory. Nucl. Phys. B238, 125-141 (1984); Non-standard 2D critical statistical models from Liouville theory. Nucl. Phys. B257 [FS14], 59-76 (1985)

28. Feigin, B. L., Fuchs, D. B.: Invariant skew-symmetric differential operators on the line and Verma modules over the Virasoro algebra. Funct. Anal. Appl. 16, 114-126 (1982); Verma modules over the Virasoro algebra. Funct. Anal. Appl. 17, 241-242 (1983); Representations of the Virasoro algebra. In: Topology, Proceedings, Leningrad 1982. Faddeev, L. D., Mal'cev, A. A. (eds.). Lecture Notes in Mathematics, vol. 1060. Berlin Heidelberg, New York: Springer 1984

Communicated by A. Jaffe

Received February 21, 1989 